\title{
Il plurilinguismo in un corso di tedesco per immigrati italiani ultrasettantenni a Berna
}

\section{Etna Rosa Krakenberger}

\section{(2) OpenEdition}

Edizione digitale

URL: http://journals.openedition.org/esp/632

DOI: 10.4000/esp.632

ISSN: 2532-0319

\section{Editore}

Centre d'Information sur l'Éducation Bilingue et Plurilingue

\section{Edizione cartacea}

Data di pubblicazione: 1 dicembre 2015

Paginazione: 57-69

ISSN: 1127-266X

\section{Notizia bibliografica digitale}

Etna Rosa Krakenberger, « II plurilinguismo in un corso di tedesco per immigrati italiani ultrasettantenni a Berna », Éducation et sociétés plurilingues [Online], 39 | 2015, Messo online il 01 octobre 2016, consultato il 20 avril 2019. URL : http://journals.openedition.org/esp/632 ; DOI : 10.4000/esp.632 


\section{"FO WUNDERBAR SO IM APRIU KANN MAN NICHT MEHR ERWARTEN, CAPISCI?" (1) IL PLURILINGUISMO IN UN CORSO DI TEDESCO PER IMMIGRATI ITALIANI ULTRASETTANTENNI A BERNA}

\section{Etna Rosa KRAKENBERGER}

In diesem Artikel möchten wir einen Blick in die Sprachenvielfalt eines besonderen Deutschkurses werfen. Es handelt sich dabei um einen Kurs für pensionierte italienische Migrantinnen in Bern. Die Teilnehmerinnen, alle älter als 70 Jahre, mit meist geringer Schulbildung, haben sich dazu entschlossen, nach der Pensionierung die Sprache des Gastlandes zu erlernen oder ihre Kompetenzen zu verbessern. Wie frühere Studien zeigen konnten, hatten viele Immigranten der 60er Fahre in der Schweiz auf Grund der Diglossie (Werlen 2007) Probleme die deutsche Standardsprache zu erlernen oder sie haben keine der Varietäten gelernt weil Zeit, Motivation und Angebot fehlten. Ein weiteres Resultat dieser diglossischen Situation, mit welcher die Migranten konfrontiert sind, ist ihre Sprachkompetenz, welche als Varietätenkontinuum zwischen einem Deutschschweizer Dialekt und der Standardsprache betrachtet werden kann. Daher werden wir anhand einiger Unterrichtssequenztranskriptionen die sprachlichen Varietäten von vier Kursteilnehmerinnen analysieren und die Funktion der verschiedenen Sprachen und Sprachvarietäten innerhalb des Kurses bestimmen.

Schlüsselwortern: Alter, Diglossie, Mehrsprachigkeit, Migration, Varietätenkontinuum, Zweitspracherwerb

Cet article explore le contexte multilingue d'un cours d'allemand conçu pour des travailleurs immigrés italiens vivant à Berne (Suisse). Les participants, tous ages de plus de 70 ans et don't la plupart n'avaient jamais étudié, ont decide d'apprendre ou d'améliorer leurs competences dans la langue de leur pays d'accueil après la retraite. Comme des etudes ont déjà montré, la plupart des migrants italiens arrives dans les années 60 avaient du mal à acquérir l'allemand standard à cause de la situation de diglossie, ou n'ont jamais appris une variété allemande par manque de temps, de motivation et de possibilités. Un autre effet de la situation linguistique des migrants à Berne est le continuum entre les variétés du dialecte suisse-allemand et l'allemand standard. Nous étudions donc les variétés langagières de quatre participants et déterminerons les fonctions des diverses langues et variétés de langues dans la classe.

Mots-clés: acquisition, continuum de variétés, diglossie, troisième âge, migration, plurilinguisme

The present paper aims at investigating the multilingual setting of a particular German language course designed for retired, established Italian workforce migrants living in the city of Berne, Switzerland. The participants, all over the age of 70 and mostly unfamiliar with learning environments, decided to learn or improve their competences in their host country's language after retirement. As previous studies have shown, most of the Italian migrants who arrived in the 1960 s had difficulties with the acquisition of standard German due to the diglossic situation (Werlen 2007) or never even learned any of the German varieties due to the lack of time, motivation and possibilities. Another outcome of the linguistic situation the migrants are confronted with in Berne, is the continuum of varieties between the Swiss-German dialect and standard German. We will therefore investigate the language varieties of four participants and determine the functions of the different languages and language varieties within the classroom.

Key-zords: age, continum of varieties, diglossia, migration, multilingualism, second language acquisition 
"Jo wunderbar so im apriu kann man nicht mehr erwarten, capisci?"

E. R. KRAKenberger
I 1 corso di lingua di cui parleremo nel seguente contributo presenta diverse particolarità. Si tratta in primo luogo di un corso che appartiene al gruppo dei cosiddetti corsi $50+$, quindi un'offerta per persone di età avanzata (vedi anche Krakenberger 2014). Questa tipologia di corsi di lingua sta guadagnando importanza negli ultimi anni a causa dei cambiamenti demografici e della crescente importanza del cosiddetto lifelong learning (vedi per esempio il programma europeo LLP: "Lifelong Learning Programme"). Nonostante queste offerte formative siano rivolte a tutta la popolazione, la maggior parte dei corsi è concepita per persone istruite, di una classe medio-alta. Diversa è la situazione del corso di cui parleremo nel presente articolo. Innanzitutto, nel caso presente si dovrebbe parlare piuttosto di un corso $70+$ poiché le signore che lo frequentano sono tutte ultrasettantenni. In effetti, il corso si svolge all'interno del Club70, il punto d'incontro interculturale per pensionati, organizzato dalla chiesa cattolica di Berna. Inoltre le partecipanti possiedono un grado d'istruzione differente dal partecipante standard di un corso di lingua: poche di loro hanno terminato le scuole medie e la maggior parte ha frequentato soltanto alcune classi della scuola elementare. Le quindici donne che hanno frequentato tale corso negli anni del nostro studio (2010-2013) sono di nazionalità italiana, immigrate negli anni '60, e con esperienze lavorative nella ristorazione, nella sartoria, in fabbriche o come donne delle pulizie. La maggior parte di loro frequenta però soltanto ora, da pensionate, un corso di lingua (tedesca) del paese ospitante (la Svizzera). Avendo vissuto nel territorio germanofono della Svizzera si sono trovate di fronte alla tipica diglossia locale, spesso definita 'mediale' (cfr. Kolde 1981), con la difficoltà riconosciuta dalle partecipanti, che nel mondo lavorativo e nella vita quotidiana in generale si parla principalmente in un dialetto svizzero tedesco mentre in classe si insegna la lingua standard.

Può sorgere la domanda sul perché delle persone che vivono da più di quarant'anni in un paese scelgano di frequentare un corso di lingua del paese ospitante soltanto da pensionati. Molte di loro raccontano che non hanno avuto bisogno delle competenze di tedesco durante la vita lavorativa perché o hanno lavorato in un ambiente dove la maggior parte dei colleghi parlava italiano, oppure hanno imparato un po' di dialetto bernese durante le interazioni con i colleghi di lavoro, riuscendo così a interagire in modo sufficiente per la vita quotidiana. Inoltre molte di loro non hanno avuto in precedenza il tempo di frequentare un corso di lingua, impegnate com'erano tra lavoro, casa e figli. Nel caso in 
"Jo wunderbar so im apriu kann man nicht mehr erwarten, capisci?"

E. R. KRAKenberger cui ci sia stata la necessità di scrivere qualcosa o di capire materiale scritto (quindi chiaramente in lingua standard), hanno poi potuto ricorrere all'aiuto da parte dei figli, che avendo frequentato le scuole in Svizzera sono di conseguenza bilingui. Data la lunga tradizione di studi sulla situazione degli immigrati italiani in Svizzera non approfondiremo qui la descrizione di essa, ma rimandiamo a opere come Rovere (1977), Haller (2004) o Moretti (2005). Le motivazioni che hanno indotto le signore a frequentare il corso da pensionate sono principalmente sociali e non meramente linguistiche: la voglia di poter passare del tempo assieme ad amiche con la motivazione di uscire di casa e scambiare esperienze durante il corso. Il corso risponde così in parte pure alla necessità di dare una struttura al tempo libero guadagnato con il pensionamento, con la speranza supplementare di imparare comunque un poco della lingua tedesca.

In un corso particolare (dal punto di vista delle partecipanti, delle loro motivazioni e della situazione linguistica che lo circonda) come quello di cui parliamo qui, anche l'uso delle lingue o delle varietà di lingue è particolare ed è ciò che vorremmo approfondire di seguito, analizzando le varietà usate da parte di quattro partecipanti (che portano gli pseudonimi Barbara, Chiara, Gabriella e Lisa).

Prima di passare alle analisi delle varietà linguistiche, offriamo un breve accenno alla vita delle quattro partecipanti. Le informazioni biografiche delle signore qui prese in considerazione derivano da interviste semistrutturate condotte nel 2013. Le storie di queste quattro donne si possono suddividere in due gruppi che mostrano alcune somiglianze tra di loro: da un lato le storie di Barbara e Chiara e dall'altro quelle di Gabriella e Lisa. Sia Barbara che Chiara hanno frequentato la scuola fino alla quinta elementare; entrambe hanno seguito i rispettivi mariti che sono partiti prima di loro per la Svizzera, Barbara all'età di 18 anni e Chiara all'età di 26 anni. In Svizzera, entrambe hanno avuto tre figli che hanno mandato alla scuola della missione cattolica. Per quanto riguarda la scuola dei propri figli e l'interazione con gli insegnanti quindi non hanno avuto bisogno di imparare la lingua tedesca o il dialetto bernese. Anche al lavoro sia Barbara che Chiara non hanno imparato la lingua del paese ospitante; Barbara ha lavorato principalmente a casa come sarta mentre Chiara ha lavorato come donna delle pulizie in un team composto da persone di varie nazioni con poche interazioni con Svizzeri. Le altre due partecipanti hanno una formazione migliore, infatti, entrambe hanno frequentato le scuole fino alla terza media. Al contrario delle 
"Jo wunderbar so im apriu kann man nicht mehr erwarten, capisci?"

E. R. KRAKenberger prime, Gabriella e Lisa sono emigrate contemporaneamente al marito in Svizzera e hanno lavorato inizialmente negli stessi posti come i propri mariti (Gabriella nell'ambito della sartoria e Lisa in una fabbrica). Dopo la nascita dei figli sia Gabriella che Lisa hanno cambiato luogo di lavoro, Gabriella ha cominciato a lavorare in un teatro come sarta e guardarobiera e Lisa ha iniziato un lavoro come donna delle pulizie all'università. Questi due lavori hanno portato a un maggiore contatto con persone tedescofone, per Gabriella anche con collaboratrici e cantanti provenienti dalla Germania. Così entrambe hanno potuto acquisire delle competenze linguistiche, Lisa piuttosto di dialetto bernese e Gabriella anche di tedesco standard. Al contrario di Barbara e Chiara esse hanno avuto bisogno pure di competenze linguistiche per la comunicazione con gli insegnanti dei propri figli poiché questi ultimi hanno frequentato la scuola svizzera e non quella della missione cattolica.

Le due situazioni differenti qui descritte - lavoro maggiormente con italofoni o a casa e bambini alla scuola della missione cattolica us lavoro a contatto con tedescofoni e bambini alla scuola svizzera - hanno portato anche a competenze linguistiche differenti, come vedremo in seguito.

Barbara e Chiara, le due donne che hanno avuto poco contatto durante la loro vita lavorativa con persone tedescofone, mostrano conseguentemente competenze minori nelle varietà di tedesco rispetto alle loro compagne. Mentre la varietà di Barbara è improntata sul dialetto svizzero tedesco, quella di Chiara è più tendente verso il tedesco standard. Analizzeremo qui di seguito le loro varietà sulla base di alcuni estratti esemplari tratti da tre lezioni tenutesi tra ottobre 2012 e febbraio 2013.

La varietà di Barbara è quella meno avanzata e la partecipante si scusa sempre con lunghi discorsi in italiano per la sua non competenza (3):

Barbara: ma di che cosa devo parlare/

Ins: $\quad$ di che cosa deve parlare . allora la tematica è bleiben oder zurückkehren cioè rimanere in svizzera o (rimanere o ritornare) zurückkehren ritornare in italia. che cosa pensa del (ritornare) ritornare del rimanere perché vorrebbe ritornare perché vorrebbe rimanere e tutto auf deutsch(in tedesco)

Barbara: eh ma io non mi so spiegare bene

Ins: ci provi

Lisa: fa niente

Ins: $\quad$ non ci sono problemi

Barbara: io mi preoccupo che così. ogni tanto qualche parola che la 
"Jo wunderbar so im apriu kann man nicht mehr erwarten, capisci?"

E. R. KRAKenberger conosco che la so non sono capace subito a . forse se mi trovo da qualche posto qualche parte magari so spiegarmi . ma però in momento quando così non capisco .. allora .. ig . zurück . italia . etza . nume (io . indietro . italia . ora . non più)

Ins: $\quad$ nicht mehr. jetzt möchten sie nicht mehr nach italien (non più . ora non vorrebbe più tornare in italia)

Barbara: immere bliibe da svizzera

(sempre rimanere/rimango qui svizzera)

Nelle poche frasi che Barbara pronuncia nella sua varietà di tedesco si può notare un'ellissi del verbo andare (in tedesco gehen) nella prima frase "ig zurück italia etza nume", e un'ellissi del soggetto (prima persona singolare $i c h$ ) nella seconda frase "immere bliibe da svizzera". Per quanto riguarda il continuum di varietà, nel presente estratto si notano quattro parole che sono da attribuire al dialetto bernese (per tutti i riferimenti che riguardano il dialetto bernese cfr. Marti 1985): il pronome della prima persona singolare $i g$; l'avverbio etza, corrispondente al tedesco jetzt ("adesso") che in bernese è pronunciato più spesso come itze, ma in altri dialetti esiste anche la forma usata da Barbara; nel caso di nume si tratterà di una forma di nicht mehr ("non più") che in bernese sarebbe nümm(e) e dove si nota il problema della pronuncia dell'umlaut [y]. Infine la forma verbale bliibe (tedesco: bleiben) che ha il tratto tipico della vocale $i$ lunga, rimasta come nel medio-alto tedesco che in tedesco standard si è invece dittongata. La forma verbale bliibe può sia rappresentare l'infinito come la prima persona singolare o la prima persona plurale e non possiamo essere sicuri di quale forma si tratti nell'enunciato di Barbara. Zurück ("indietro") è invece chiaramente tedesco standard poiché in bernese sarebbe zrügg. Immere ("sempre") e da ("qui") sono forme che potrebbero appartenere a entrambe le varietà (sia bernese che tedesco standard), con però l'aggiunta della vocale finale in immer. Inoltre, negli enunciati maggiormente tedescofoni Barbara mantiene la forma italiana quando indica i nomi di luogo (italia, svizzera). Possiamo quindi concludere che la sintassi di Barbara è un sintassi che funziona perlopiù per semplici giustapposizioni, la morfologia verbale è estremamente semplificata e alcune parole sono mantenute nella lingua madre (L1). In questo breve estratto quattro parole su sette sono da attribuire al dialetto bernese, due possono essere di entrambe le varietà e solo una parola (zurück) è chiaramente da ascrivere al tedesco standard. Quest'ultima potrebbe anche derivare da un effetto di priming (2) poiché poco prima l'insegnante parla di zurückkehren. 
"Jo wunderbar so im apriu kann man nicht mehr erwarten, capisci?"

E. R. Krakenberger
La seconda partecipante con una varietà semplificata (l'uso del termine varietà semplificata si rifà allo studio di Zanovello-Müller (1998); torneremo più avanti allo schema da lei sviluppato) è Chiara:

Chiara: ja ig eh fufezig eh jaare vorhere eh verheiratet

(si io eh cinquanta eh anni prima eh sposata)

. sposata no/

Ins: verheiratet

(sposata)

Chiara: $\quad$ ibliibe in italia nagare drai monate eh mini mann ist

(io rimango in italia poi tre mesi eh mio marito è)

da in schwaiz

(qui in svizzera)

Ins: $\quad$ in die schweiz gekommen

(venuto in soizzera)

Chiara: eh ig eh. gomo in schwaiz

(eh io eh. vengo in svizzera)

Ins: $\quad$ zu ihrem mann sind sie gekommen

(è venuta da suo marito)

Chiara: ja. eh nagare eh zwe jaare ig habe kinder krige

(si. eh poi eh due anni io ho avuto bambini ottenere)

Ins: nach zwei jahren haben sie die kinder bekommen (dopo due anni ha avuto i figli)

Chiara: kinder bekomme eh mini eh mini elter gesunde nid gut (avuto figli eh miei eh i miei genitori salute non bene)

Ins: $\quad$ ah sie waren krank

Chiara: ja (ah erano malati)

(si)

Le semplificazioni si notano per esempio a livello della sintassi con l'ellissi del participio gewesen nella frase "mini mann ist da in schwaiz". Per esprimere il tempo passato Chiara non si serve della morfologia verbale ma applica indicazioni come nagare drai monate (usando maggiormente la forma del presente indicativo come blibe o ist). Anche a livello lessicale ricorre all'uso di forme analitiche come per esempio gesunde nid gut (salute non bene) al posto del aggettivo krank. Per quanto riguarda le varietà del tedesco si osservano parole tratte dal dialetto, come abbiamo già osservato in Barbara, come il verbo bliibe o il pronome della prima persona singolare $i g$. Inoltre si notano vocaboli non ancora presenti nel repertorio di Barbara come nagare (che in tedesco sarebbe nachher e significa "dopo"), la particella di negazione nid (in tedesco nicht), il numero zwe (in tedesco zwei) o il pronome possessivo di prima persona mini 
"Jo wunderbar so im apriu kann man nicht mehr erwarten, capisci?"

E. R. KRAKenberger (in tedesco mein). Quest'ultimo non viene accordato con il genere da Chiara; così mini, che nelle varietà di nativi rappresenta la forma femminile singolare o le forme plurali di tutti e tre i generi (Marti 1985: 99), viene sovraesteso da Chiara anche a marca del maschile singolare come in mini mann ("il mio marito/uomo"). Incontriamo comunque più forme tendenti verso la lingua standard rispetto al repertorio di Barbara come jaare, verheiratet, drai monate, mann ist da in schwaiz, habe kinder krige, kinder bekomme e elter gesunde. Si nota anche che, nonostante la varietà di Chiara sia semplificata, essa è comunque più elaborata rispetto alla varietà di Barbara. Considerando le loro biografie presentate poc'anzi, non troviamo una spiegazione per questa divergenza, poiché entrambe hanno potuto fare a meno della lingua tedesca durante la vita lavorativa e nelle interazioni riguardanti l'ambito scolastico dei propri figli. La differenza è da ricercare piuttosto in una diversa motivazione. Infatti, Barbara frequenta il corso principalmente perché una sua amica l'ha convinta a venire per stare in compagnia, mentre Chiara ha più intenzione di migliorare le competenze linguistiche e racconta che porta sempre con sé un libro con vocaboli annotati per poterli studiare in momenti di attesa, per es. dal medico o in situazioni simili. Inoltre afferma di voler raggiungere una certa indipendenza dai propri figli per questioni amministrative, mentre nel caso di Barbara sono proprio i figli a sottolineare che secondo loro questo corso di tedesco non ha senso, dicendo (estratto dell'intervista con Barbara):

"Mamma, ma chi te lo fa fare andare a fare questo corso di tedesco? A che ti serve?"

Con lo stesso argomento della motivazione possiamo anche spiegare la differenza delle tendenze: Barbara tende più verso il dialetto mentre la varietà di Chiara tende più verso la lingua standard. Poiché Barbara è poco motivata a studiare, ha imparato probabilmente le poche espressioni che usa in modo spontaneo già durante la vita lavorativa e di conseguenza sono più spesso tendenti verso il dialetto svizzero tedesco. Chiara invece migliora le proprie competenze attivamente frequentando il corso di tedesco e studiando inoltre al di fuori del corso, applicando quindi maggiormente anche elementi della lingua standard.

Le altre due partecipanti prese in considerazione (Gabriella e Lisa) hanno avuto più contatto con tedescofoni durante la loro vita lavorativa. Mentre Lisa ha acquisito principalmente competenze di dialetto svizzero tedesco, Gabriella è entrata in contatto anche con tedescofoni della Germania e ha sviluppato così una varietà tendente maggiormente verso il tedesco standard. Come 
"Jo wunderbar so im apriu kann man nicht mehr erwarten, capisci?"

E. R. KRAKenberger potremo osservare negli estratti seguenti, il contatto con la lingua ha favorito in entrambe lo sviluppo di competenze più elaborate rispetto a quelle che abbiamo potuto osservare negli estratti di Barbara e Chiara:

Gabriella :ich habe nur eine tochter

(io ho soltanto una figlia)

Ins: und sieben enkelkinder/

Gabriella: nei moment ich habe drai ((ridono)) viele leute hatten (no aspetta io ho tre ((ridono)) tanta gente aveva) siben kinder . nei will sagen nur ich habe drai kinder (sette figli . no voglio dire solo io ho tre figli) zwe bub und ain mädchen und di mädchen ist in sizilia (due maschi e una femmina e la femmina è in sicilia)

Ins: das mädchen ist in sizilien (la ragazza è in sicilia)

Gabriella: verhairatet mit di kinder und ich habe gern wenne hir (sposata con i figli e mi piace se era qui) war und nid in sizilie (e non in sicilia)

Notiamo che ci sono meno semplificazioni lessicali, le difficoltà si mostrano invece a livelli più avanzati della morfosintassi come nella frase che richiede la forma del periodo ipotetico "ich habe gern wenne hir war". La forma corretta sarebbe ich hätte gern wenn sie hier wäre, Gabriella usa invece l'indicativo presente habe e l'imperfetto war. Nella frase nei will sagen nur osserviamo un'ellissi del soggetto, probabilmente come influenza della L1 dove una frase come no, voglio dire è consentita poiché l'italiano è una lingua prodrop, mentre in tedesco il soggetto, quindi in questo caso il pronome della prima persona singolare $i c h$, è obbligatorio. Si osservano comunque anche problemi con il genere (di mädchen al posto di das mädchen), o con la marcatura del caso come in mit di kinder che dovrebbe essere mit den kindern, quindi richiede il dativo mentre Gabriella usa la forma che corrisponde al nominativo e all'accusativo. Un ulteriore indizio della maggiore inclinazione al tedesco standard della varietà di Gabriella è per esempio l'uso dell'imperfetto hatten che in dialetto svizzero tedesco non esiste (le uniche forme del passato presenti nel dialetto sono il Perfekt ed il Plusquamperfekt). Chiaramente di forma dialettale sono soltanto le particelle di negazione nei e nid come anche il numero zwe (in tedesco zwei). I nomi di paesi/regioni sembrano variare spesso all'interno degli enunciati, così Gabriella usa sizilia (forma ibrida tra svizzero tedesco e italiano) e poi sizilie (svizzero tedesco) proba- 
"Jo wunderbar so im apriu kann man nicht mehr erwarten, capisci?"

E. R. Krakenberger bilmente su influsso dell'introduzione della forma tedesca (sizilien) da parte dell'insegnante. Nell'estratto di Gabriella la maggior parte del lessico e anche molte strutture morfosintattiche sono tratte quindi dalla lingua standard al contrario dell'esempio che vediamo qui sotto di Lisa:

Lisa: $\quad$ i verste aus aber wäg redä isch schwirig

(io capisco tutto ma per parlare è difficile)

Ins: $\quad$ selber zu sprechen ist schwieriger natürlich

(parlare autonomamente è difficile certo)

Lisa: $\quad$ es isch verukt

(è pazzesco)

$[\ldots]$

Lisa: $\quad$ wenn mann hat umfall gha . eh nach zeङ nache aina (quando marito ha avuto incidente . eh dopo diङ dopo una) wocha . er isch in koma gsi und nid wachä und aina tag (settimana . lui è stato in coma e non svegliano e un giorno) isch zä arzt im insuspital (è dieci medici nell'ospedale (di Berna))

Ins: $\quad$ zehn ärzte (dieci medici)

Lisa: $\quad z \ddot{a} . n i i$ italiänisch gredt ni (dieci . mai italiano parlato mai)

Ins: $\quad$ keiner hat italienisch gesprochen (nessuno ha parlato italiano)

Lisa: $\quad$ u när u nächär $i$ ha frage er hat gesagt nai tut mir (e poi e poi io ho chiesto lui ha detto no mi dispiace) laid frau Lisa kann nid italienisch (signora Lisa non so italiano)

La varietà di Lisa è più semplificata rispetto a quella di Gabriella ma chiaramente tendente verso il dialetto bernese. Come semplificazione possiamo evidenziare per esempio nella frase "nii italiänisch gredt ni" la sovraestensione della particella di negazione nicht (nell'esempio più simile alla forma dialettale nid) a luoghi in cui sarebbe richiesto kein. Inoltre osserviamo un caso di sovraestensione di wenn all'ambito di als (in italiano entrambe le forme sono realizzate da quando) nell'esempio "wenn mann hat umfall gha". Anche le forme verbali sono talvolta semplificate come wachä che dovrebbe essere verwachät (in tedesco erwacht), o "i ha frage" al posto della forma corretta del participio "i ha gfragt". Comunque in altri casi la forma del participio è conosciuta sia nella forma dialettale (gha, gsi, gredt) sia in quella della lingua standard (gesagt). Inoltre si osservano un'ellissi del soggetto $i c h$ in "kann nid italienisch" e un'ellissi del participio gsi in "isch zä arzt im 
"Jo wunderbar so im apriu kann man nicht mehr erwarten, capisci?"

E. R. KRAKenberger insuspital". La tendenza verso il dialetto bernese viene evidenziata dal fatto che si riscontrano poche forme ascrivibili solamente alla varietà del tedesco standard. Un esempio di varietà più tendente verso lo standard è il discorso indiretto (ultima riga) dove Lisa riporta le parole di un medico tedescofono. In questo caso la partecipante usa prevalentemente la lingua standard a parte la particella di negazione nid. L'uso di un lessico più tedesco qui si nota anche nella forma di italienisch per la quale poco prima viene usata la forma dialettale italiänisch. Le restanti parole ascrivibili alla varietà standard tedesca sono sparse in tutto il discorso senza motivazioni apparenti e potrebbero spesso anche essere forme del dialetto come verste o wenn man hat umfall. La maggior parte del lessico è invece chiaramente tendente verso il dialetto come le forme verbali redä (in tedesco reden), isch (in tedesco ist), gha (in tedesco gehabt), gsi (in tedesco gewesen) e gredt (in tedesco geredet), ma anche altre parole come gli avverbi verukt e nachär (in tedesco verrückt $\mathrm{e}$ nachher/danach) o il numero zä (in tedesco zehn). Ancora più marcato verso il dialetto bernese è l'uso della vocalizzazione di [1] che diventa [u] (vedi Marti 1985: 55-58). Nell'estratto qui presentato ne troviamo due casi: aus che deriva da alles e insuspital al posto di inselspital. Lisa è l'unica tra le partecipanti del corso ad applicare questa regola fonetica del bernese, probabilmente perché è anche la partecipante che ha avuto maggiore contatto con gli abitanti del luogo.

Se volessimo inserire ora le varietà maggiormente usate di queste quattro parlanti in uno schema come è stato proposto da Zanovello-Müller (1998: 182), dove l'autrice distingue tra l'asse di semplificazione e l'asse della varietà, otterremmo approssimativamente il seguente quadro: Gabriella si trova sull'asse del 'poco semplificato' e del 'tedesco' mentre Chiara si sposta verso la parte più semplificata. Lisa sta tra Gabriella e Chiara per quanto riguarda l'asse della semplificazione ma tendente chiaramente più verso la varietà di svizzero tedesco. Barbara invece si trova al lato opposto di Gabriella quindi sulla posizione di una semplificazione maggiore, tendente verso lo svizzero tedesco.

Come vedremo tra poco la stessa persona può però muoversi durante l'interazione all'interno del continuum di varietà e non solo di varietà tedesche ma anche di varietà italiane. Non sempre è evidente quanto le persone siano però coscienti della differenza tra dialetto e lingua standard, nonostante spesso il cambiamento verso la lingua standard sia indotto dalla situazione del corso dove l'insegnante cerca di rilevare le differenze tra le varietà. Come abbiamo visto per esempio nel caso di italiänisch e italienisch, all'interno di una conversazione si possono incon- 
"Jo wunderbar so im apriu kann man nicht mehr erwarten, capisci?"

E. R. Krakenberger trare diverse forme per la stessa parola, talvolta anche ibride. Nel prossimo estratto osserviamo una gamma maggiore di varietà che passa dal dialetto italiano, all'italiano, a forme improntate dal tedesco standard fino al dialetto svizzero tedesco. Così lo stesso concetto di "essere svizzera" viene espresso da Lisa prima in una varietà tendente al dialetto italiano te sei scivizzera, poi in italiano tu sei svizzera. In seguito passa al tedesco standard con la frase Piera hat gesagt per servirsi poco dopo di una varietà tendente verso il dialetto svizzero tedesco si isch schwizeri.

Lisa: $\quad$ te sei scivizzera . tu sei svizzera . Piera hat gesagt

(Piera ha detto) $i$ scho .

(io si.)

Ins: $\quad$ sie wollten gerne zurück kehren/ aber sie ist an der

(lei voleva volentieri tornare / ma è al confine con) grenze zur schweiz oder/

Lisa: $\quad$ ja si isch valtellina

(si lei è valtellina)

Ins: genau .

(esatto .)

Lisa: $\quad$ si isch schwizeri

(lei è soizzera)

Come abbiamo potuto osservare sia nelle sequenze di Barbara ma anche in quella di Lisa ci può essere una larga presenza della lingua italiana. Questa presenza della L1 delle partecipanti nel corso di tedesco deriva da un lato dai diversi livelli di competenza (che richiedono talvolta delle spiegazioni da parte dell'insegnante in italiano) e dall'altro dalla motivazione sociale, che talvolta induce le partecipanti a parlare delle proprie esperienze raccontando dei lunghi episodi in italiano senza tener conto dei tentativi da parte dell'insegnante di reintrodurre il tedesco (giacché si tratta di un corso di tedesco):

Gabriella: adesso suona/

Lisa: no

Gabriella: ah peccato

Lisa: sì perché poi era troppo italiano eh flauto che le piaceva il piano poi cominciava l'inglese e per questo per quello

Ins: $\quad$ es war zu viel mit italienisch englisch der flöte (era troppo con italiano inglese il flauto)

Lisa: $\quad$ sì sì tutto le piaceva lo sport era troppo . dieci undici anni però cinque anni di conservatorio ha fatto

Ins: $\quad$ fünf jahre

(cinque anni) 
"Jo wunderbar so im apriu kann man nicht mehr erwarten, capisci?"

E. R. KRAKenberger

\section{BIBLIOGRAFIA:}

Lisa:

sì e mo sa suonare bene

Anche se le analisi potrebbero essere più approfondite siamo riusciti a constatare che il corso di tedesco per signore italiane pensionate a Berna è uno spazio multilingue. In questo spazio le partecipanti, le cui competenze linguistiche sono alquanto divergenti e si situano in vari punti sull'asse della semplificazione, usano un continuum di varietà (cfr. Dal Negro 1993) tra un dialetto svizzero tedesco e il tedesco standard dovuto ai vari modi e alle varie situazioni di apprendimento spontaneo. Oltre alle varietà tedescofone si osservano varietà di italiano talvolta cariche di regionalismi e dialettismi (vedi l'esempio di Lisa). L'insegnante cerca invece di usare la lingua standard in entrambi i casi, il tedesco per le esercitazioni e l'italiano per le spiegazioni di concetti grammaticali e altre parti dell'insegnamento. La grande varietà linguistica è anche dovuta al fatto che si tratta di un luogo principalmente di incontro dove l'apprendimento linguistico passa in secondo piano e l'italiano domina. Nonostante ciò si è potuto notare un aumento delle competenze in tutte le partecipanti, se non a livello meramente grammaticale almeno su quello comunicativo. La frequentazione del corso ha portato le partecipanti (spesso confrontate con stereotipi e rimproveri per non aver acquisito maggiori competenze della lingua del paese ospitante nonostante la lunga permanenza) a superare il disagio e la paura di servirsi attivamente delle loro varietà di tedesco nell'uso quotidiano (vedi anche Krakenberger 2015).

DAL NEGRO S. 1993. Il tedesco degli immigrati a Zurigo. Milano: Guerini Studio.

DIETH E. 1986. Schweyzertütschi Dialäktschrift: Dieth-Schreibung. (2) Aarau und Frankfurt am Main: Verlag Sauerländer.

HALLER E. (Ed.) 2004. Gli italiani in Syizzera: un secolo di emigrazione. Bellinzona: Casagrande.

KOLDE G. 1981. Sprachkontakte in gemischtsprachigen Städten: vergleichende Untersuchungen über Voraussetzungen und Formen sprachlicher Interaktion verschiedensprachiger jugendlicher in den schweizer Städten Biel/Bienne und Fribourg/Freiburg. Wiesbaden: Franz Steiner.

KRAKENBERGER E. R. 2014. "50+L2": una formula utile dopo il pensionamento? 115-130 in BERTHELE R. e KAISER I. (Eds.) Mehrsprachigkeit und Lebensalter. Neuchâtel: Institut de linguistique de l'Université, 99.

KRAKENBERGER E. R. 2015. Pensionamento e plurilinguismo. Un'indagine qualitativa su immigrati italiani e su svizzeri tedeschi. Ospedaletto: Pacini.

MARTI W. 1985. Berndeutsch-Grammatik. Bern: Francke Verlag. MORETTI B. 2005. Il laboratorio elvetico, pp. 15-79 in MORETTI 
"Jo wunderbar so im apriu kann man nicht mehr erwarten, capisci?"

E. R. Krakenberger

\section{SITO INTERNET:}

\section{NOTE}

(1) ,fo wunderbar so im apriu kann man nicht mehr erwarten, capisci?"“ può essere tradotto con „sì ottimo così in aprile non ci si può aspettare di più, capisci?" ed è un esempio del multilinguismo presente non soltanto all'interno del corso in generale ma delle varietà presenti nel repertorio delle parlanti: in corsivo sono segnalate le espressioni chiaramente ascrivibili a un dialetto svizzero tedesco e accanto a espressioni del tedesco standard appaiono anche parole italiane.

(2) Ins. sta per insegnante, mentre le partecipanti sono indicati con i corrispettivi pseudonimi. Nelle trascrizioni degli enunciati in tedesco da parte dell'insegnante viene usata la grafia standard mentre per la trascrizione degli enunciati nel continuum di varietà delle partecipanti (segnalate in corsivo) è stata usata la norma di trascrizione della cosiddetta ,weite-Dieth-Schreibung “" (Dieth 1986). Per le espressioni non italiane è stata inserita inoltre una traduzione interlineare indicata tra parentesi. Con [. ] viene indicata una pausa, mentre [ / ] indica un’intonazione ascendente.

(3) Per priming intendiamo il fatto descritto nella psicologia che uno stimolo può influenzare l'elaborazione di un prossimo elemento, nel nostro caso la presenza della parola zurückkehren può, in modo inconscio, aumentare la probabilità dell'uso della stessa parola da parte dell'interlocutore (Per una descrizione più approfondita del priming strutturale rimandiamo a Pickering e Ferreira 2008). 